
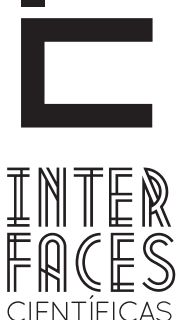

SAÚDE E AMBIENTE

\title{
PERFIL EPIDEMIOLÓGICO E FUNCIONAL DE PACIENTES COM DISTROFIA MUSCULAR DE DUCHENNE ATENDIDOS EM CLÍNICAS DE FISIOTERAPIA NA CIDADE DE ARACAJU
}

Mayanna Machado Freitas ${ }^{1}$ Allen Rafael Cardoso Santos ${ }^{1}$
Juciele Valéria Ribeiro de Oliveira ${ }^{2}$ Daniela da Costa Maia²

\section{RESUMO}

O presente artigo trata da Distrofia Muscular de Duchenne que é a mais comum e também a mais grave das distrofias musculares, onde os sinais e sintomas são mais severos com evolução mais rápida, podendo o óbito ocorrer por volta da $2^{a}$ ou $3^{a}$ década de vida. Atualmente, a incidência está estimada em torno de um para cada três mil e quinhentos nascidos vivos do sexo masculino. Os objetivos deste artigo são: conhecer a prevalência de Distrofia Muscular de Duchenne no município de Aracaju-SE, bem como caracterizar o perfil funcional apresentado por estes pacientes. A população de estudo compreendeu todos os pacientes com Distrofia Muscular de Duchenne, atendidos nas clínicas de fisioterapia desta cidade. Os dados foram coletados no período de janeiro a abril de 2011, inicialmente nos prontuários de cada paciente, e posteriormente, os pacientes identifica- dos foram submetidos à avaliação clínico epidemiológica. Dentre as limitações funcionais encontradas na maioria dos pacientes pesquisados, inclui principalmente o comprometimento de membros inferiores. Verificou-se que os pacientes avaliados no município de Aracaju apresentaram comprometimento funcional moderado para a realização das atividades de vida diária, caracterizando a necessidade de implantação de ações e políticas privadas e públicas voltadas para melhoria na qualidade de vida desses pacientes.

\section{PALAVRAS-CHAVE}

Fisioterapia. Distrofia Muscular de Duchenne. Epidemiologia 


\section{ABSTRACT}

The Duchenne Muscular Dystrophy is most common and the most severe of the muscular dystrophies, where the signals and symptoms are more severe with faster evolution, death can occur around the 2nd or 3 nd decade of life. Currently, the incidence is estimated at around one for each three thousand five hundred male births. To determine the prevalence of Duchenne Muscular Dystrophy in the city of Aracaju-SE, and to characterize the functional profile presented by these patients. The study included all patients with Duchenne Muscular Dystrophy, assisted at the physiotherapy clinics in this city. The data were collected from January to April 2011; initially in the medical records of each patient, and subsequently, the identified patients were submitted for clinical and epidemiological evaluation. Among the functional limitations found in most patients studied, mainly includes the involvement of the lower limbs. The patients evaluated in Aracaju showed moderate functional impairment to perform activities of daily living, characterizing the need to implement actions and private and public policies aimed at the improvement of life quality of these patients.

\section{KEYWORDS}

Physiotherapy. Duchenne Muscular Dystrophy. Epidemiology

\section{INTRODUÇÃO}

As miopatias são doenças que se caracterizam por alterações estruturais ou funcionais dos músculos. Dentre as miopatias, podemos citar as distrofias musculares, consideradas miopatias primárias, geneticamente determinadas, que se caracterizam clinicamente por intensa variabilidade do fenótipo,

\section{RESUMEN}

La distrofia muscular de Duchenne es la más común y la más grave de las distrofias musculares, donde los signos y síntomas son más intensos con una evolución más rápida y la muerte puede ocurrir alrededor de la $2^{\mathrm{a}}$ o $3^{a}$ década de la vida. En la actualidad, la incidencia se estima en alrededor de uno por cada três mil quinientos nacidos vivos del sexo masculino. Conocer la prevalencia de la Distrofia Muscular de Duchenne en la ciudad de Aracaju-SE, así como caracterizar el perfil funcional que presentan estos pacientes. La población de estudio consistió en todos los pacientes con distrofia muscular de Duchenne, asistidos por clínicas de terapia física en esta ciudad. Los datos fueron recogidos entre enero y abril de 2011, inicialmente en los registros clínicos de cada paciente, $y$, posteriormente, los pacientes identificados fueron sometidos a evaluación clínico-epidemiológica. Entre las limitaciones funcionales que se encontraron en la mayoría de los pacientes estudiados, se destaca el acometimiento de los miembros inferiores. Los pacientes evaluados en la ciudad de Aracaju mostraron deterioro funcional moderado para realizar actividades de la vida diaria, lo que caracteriza la necesidad de implementar acciones y políticas públicas y privadas dirigidas a mejorar la calidad de vida de estos pacientes.

\section{PALABRAS CLAVE}

Terapia Física. Distrofia Muscular de Duchenne. Epidemiología. nível de gravidade e anatomopatologicamente pelo encontro de padrão distrófico inespecífico na fibra muscular. Formam um grupo de doenças caracterizadas clinicamente por fraqueza e atrofia musculares progressivas, com grande variação quanto à intensidade do comprometimento e época do início 
das manifestações (BORGES et al., 2007; NAIR et al., 2001; REED, 2002).

A distrofia muscular do tipo Duchenne corresponde a um grupo heterogêneo de doenças de caráter hereditário e caracteriza-se por um defeito bioquímico intrínseco da célula muscular. Não são encontradas evidências clínicas ou laboratoriais de envolvimento da medula espinhal, do sistema nervoso periférico ou da junção neuromuscular (CAROMANO, 1999; FACHARDO, 2004; OTSUKA et al., 2005).

Atualmente, a incidência da DMD está estimada em torno de 1:3.500 nascidos vivos do sexo masculino. No Brasil, ocorrem por ano, cerca de 700 novos casos. Nos estudos realizados por Dooley (2010) no Canadá entre 1969 e 2008, apresentaram uma incidência estável de 1:4700 nascimentos do sexo masculino durante o período deste estudo. Embora em algumas situações possa afetar o gênero feminino na frequência de 1:2.500, a mulher possui dois cromossomos $X$, se um deles estiver afetado pelo erro genético, o outro compensa com a alteração e a doença não se manifesta, tornando esta uma portadora assintomática. A doença pode desenvolver-se excepcionalmente de forma sintomática em casos de Síndrome de Turner (45X) e quando os dois genes herdados forem afetados (BORGES et al, 2007; MACHADO, 2007; BAUMGARTNER e RUIZ, 2008; FACHARDO, 2004).

A DMD é a mais comum e também a mais grave das distrofias musculares, onde os sinais e sintomas são mais severos com evolução mais rápida, podendo o óbito ocorrer por volta da $2^{a}$ ou $3^{a}$ década de vida. Os sinais mais precoces são a marcha anserina e o sinal de Gowers, que tornam-se evidentes e lentamente progressivos a partir dos quatro anos de idade. Embora existam variações na evolução do quadro clínico, normalmente os portadores de DMD não são capazes de continuar deambulando após os 16 anos de idade (FREZZA et al., 2005; REED, 2002; OVANDO, 2008; GYPEL, 1996).
A avaliação funcional é o estudo do comportamento motor individual em determinada situação, sendo fundamental na área da saúde, pois envolvem recursos que ajudam na construção e aperfeiçoamento do tratamento. Para avaliação desta funcionalidade de maneira mais específica, existem várias escalas, dentre elas: o Índice de Barthel, as escalas de Vignos e de Brooke, escala de Spencer e Archibald e a escala Medida da Função Motora Grossa (GMFM). Para a realização deste estudo, usou-se a escala EK, Egen Klassifikation, desenvolvida na Dinamarca e validada para a língua portuguesa em outubro de 2006 por Martinez et al, com o objetivo de quantificar o grau de capacidade funcional de pacientes com Distrofia Muscular de Duchenne em fase avançada da doença. É composta por dez itens, cada um contribuindo para uma visão global da função do estágio não ambulatório de DMD (RODRIGUES, 2009; IWABE et al, 2008; CAROMANO, 1999).

A ausência de estudos epidemiológicos da Distrofia Muscular de Duchenne tem levado a não tomada de atitudes coerentes por parte dos poderes públicos e privados, das sociedades médicas e científicas. Em Sergipe, não existem estudos que tratem da epidemiologia desta doença. Este trabatho justifica-se pela necessidade de contribuir para possíveis ações mais precisas e eficazes por parte de agentes públicos e privados, que estão direta ou indiretamente envolvidos na prestação de serviços de saúde, bem como promover a formação de conhecimento crítico e humanístico que possam favorecer aos estudantes e profissionais informações verdadeiras e relevantes.

0 presente artigo teve como objetivo conhecer a prevalência de DMD no município de Aracaju-SE, bem como caracterizar o perfil funcional apresentado por estes pacientes, que se encontram em acompanhamento em clínicas de Fisioterapia na cidade de Aracaju. 


\section{METODOLOGIA}

\section{TIPO DE ESTUDO}

Trata-se de um estudo observacional, do tipo transversal e não randomizado.

\section{POPULAÇ̃̃O DE ESTUDO}

Foram incluídas todas as crianças, nascidas e atendidas nas clínicas de fisioterapia de Aracaju, com diagnóstico clínico de DMD.

\section{ANÁLISE DAS VARIÁVEIS}

0 estudo foi realizado no período de janeiro a abril de 2011 e os dados foram coletados nos prontuários de cada paciente nos períodos entre 2006 e 2011, para ter certeza do diagnóstico de DMD. Foi utilizada uma ficha de avaliação (APÊNDICE A) composta por dados pessoais do paciente como: nome completo do voluntário, cor, nome do pai ou mãe, renda familiar, se possui casa própria, se o voluntário frequenta escola, ensino superior, quando iniciou o processo de escolarização, se apresenta dificuldades na aprendizagem, se realiza marcha, idade de aparecimento do sinal de Gowers, idade de perda de marcha, se possui cadeira de rodas, controle de tronco, encurtamentos, quais as atividades nas horas de lazer e se possui amigos.

Para avaliação do perfil funcional, foi utilizada uma escala validada para a língua portuguesa, Egen

\section{COLETA DE DADOS}

Clínicas de Fisioterapia situadas na cidade de Aracaju/SE.

\section{COMITÊ DE ÉTICA EM PESQUISA [CEP]}

A pesquisa foi submetida à aprovação pelo Comitê de Ética em Pesquisa através do Parecer Consubstanciado de cadastro de número 030311 - R.

Klassifikation (ANEXO A), tendo como objetivo, medir o grau de acometimento funcional para atividades de vida diária (AVD's) de pacientes com DMD. É composta por 10 itens descritos em frases curtas e escritas de modo claro e acessível. Os pacientes foram orientados a escolher uma alternativa que fosse condizente com sua aptidão física e em casos de não soubessem responder, o cuidador poderia intervir na resposta.

A coleta de dados foi realizada pelos próprios pesquisadores e autorizado pelos responsáveis, com assinaturas do Termo de Consentimento Livre e Esclarecido, atendendo aos termos da resolução 196/96, de 10 de outubro de 1996 do Conselho Nacional de Saúde do Ministério de Saúde. Os dados serão apresentados em gráficos e tabelas em relação ao número e porcentagem dos achados estatísticos pelo Teste de Fisher.

\section{RESULTADOS}

Todos os casos identificados foram do gênero masculino, com mediana de faixa etária de 13,77 anos (Ta- bela 1). 0 mais jovem possui 4 anos e o mais velho 30 anos, sendo este último, o único que veio a óbito. 
Tabela 1: Idade da Amostra Estudada.

\begin{tabular}{|c|c|c|}
\hline Idade em anos & Quantidade & $\%$ \\
\hline 4 & 1 & $11 \%$ \\
\hline 11 & 2 & $22 \%$ \\
\hline 12 & 3 & $34 \%$ \\
\hline 16 & 2 & $22 \%$ \\
\hline 30 & 1 & $11 \%$ \\
\hline Total & 9 & $100 \%$ \\
\hline
\end{tabular}

0 surgimento do sinal de Gowers foi observado em $45 \%$ dos indivíduos com 6 anos, $22 \%$ aos 2 anos, 22\% dos 3 aos 4 anos e, $11 \%$ aos 7 anos de acordo com a Figura 1 e nesse período, as crianças deram início ao tratamento fisioterapêutico.

Figura1: Distribuição percentual dos pacientes com DMD, segundo a idade de aparecimento do sinal de Gowers.

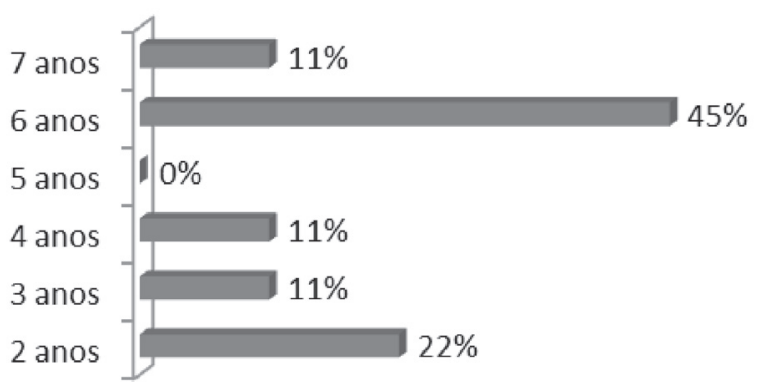

Figura 2: Distribuição percentual da situação de deambulação dos pacientes com DMD.

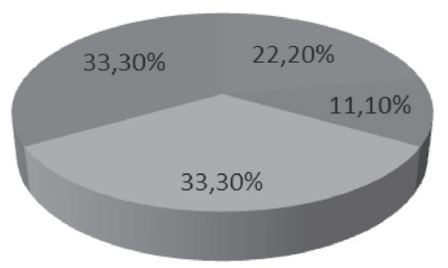

$$
\begin{aligned}
& \text { — ainda anda } \\
& \text { nunca andou } \\
& \text { n perdeu } 10 \text { anos } \\
& \text { a perdeu } 11 \text { anos }
\end{aligned}
$$

Dentre as características socioculturais e econômicas familiares destacam-se: a cor da pele predomi- nantemente parda, que seguem as características raciais regionais (IBGE, 2010); a renda familiar, que gira em torno de 2 salários mínimos; o grau de instrução dos pais é predominantemente do ensino fundamental; e $100 \%$ das famílias possuem casa própria.

A associação dos dados socioeconômicos culturais com uma das características clínicas, a exemplo do uso de cadeira de rodas, observa-se que $22,2 \%$ dos pacientes com DMD não possuem cadeira de rodas, em razão destes ainda possuírem marcha. No entanto, para os demais $66,6 \%$ dos pacientes usuários de cadeira de rodas, o tipo de cadeira usual era manual e apenas um paciente (11\%) possuía cadeira de rodas elétrica. Foi analisada a relação entre os dados de condições sócio econômicas e o nível de funcionalidade do paciente, utilizando-se como parâmetros: a renda familiar e o uso de cadeira de rodas.

Nesta análise, observou-se também que as condições socioeconômicas, associadas ao nível de escolaridade dos familiares interferem na possibilidade de acesso dos pacientes a melhores recursos terapêuticos e, consequentemente a uma melhor qualidade de vida.

Quando é investigado sobre o círculo de amizades, encontrou-se que $44,4 \%$ dos pacientes relataram possuir amigos e $55,5 \%$ demonstraram ter dificuldade de relacionamentos, conforme Figura 3. Contudo, quanto ao aspecto do lazer, observou-se que os pacientes participam de diversas atividades, conforme Figura 4.

Figura 3: Demonstração percentual do ciclo de amizades dos pacientes de DMD.

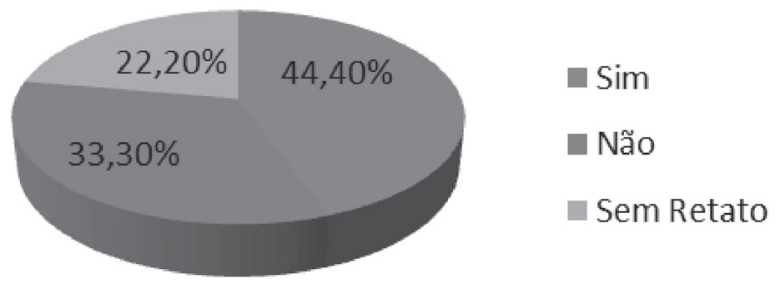


Figura 4: Atividades de lazer preferenciais eleitas pelos pacientes de DMD.

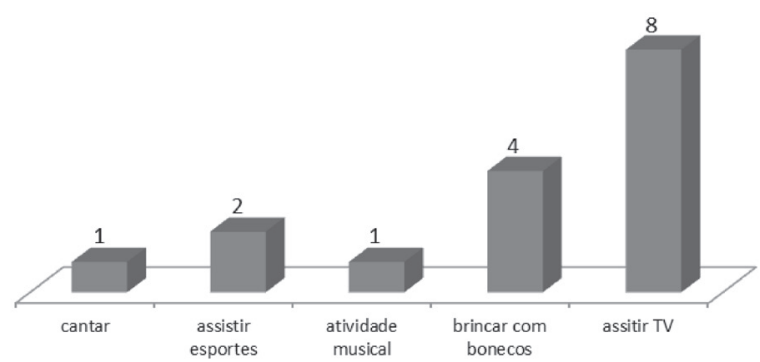

Em relação escolaridade, aprendizagem e cognição, neste estudo, encontrou-se que $90 \%$ pacientes iniciaram a escolarização entre os 4 e os 7 anos de idade, conforme Figura 5; encontrou-se também que apenas

\section{DISCUSSÃO}

Estima-se que $55 \%$ a $90 \%$ dos pacientes com DMD morrem por insuficiência respiratória entre 16 e 19 anos de idade, e raramente após os 25 anos, concordando com os dados do presente estudo, onde apenas um voluntário nesta faixa etária veio a óbito durante a realização da pesquisa (FACHARDO, 2007; PARREIRA et al., 2007).

De acordo com Santos et al. (2006), os dados variam de 13 a 35 a cada 100.000 nascidos vivos do sexo masculino, sendo raro em indivíduos do sexo feminino, ocorrendo somente em mulheres com Síndrome de Turner ou translocação X-autossômica. Para Silva et al. (2003), cerca de $1 / 3$ das mães que tem apenas um filho afetado não são portadoras de uma mutação genética da DMD. Segundo Baumgartner e Ruiz (2008), o gênero masculino prevalece em uma proporção de 1 em cada 3000 a 4.000 nascidos vivos, sendo 113 o número de portadores de DMD a cada ano na cidade de São Paulo (ARAGAO et al, 2009).

Em um estudo realizado por Peterlin et al. (1997) com a população eslovena, a incidência e prevalência de DMD são de 2.9 a cada 100.000 e 13.8 a cada
Figura 5: Distribuição percentual da idade de introdução do paciente de DMD na escota.

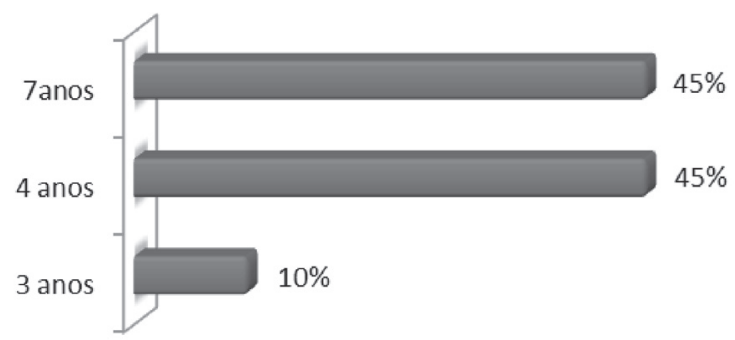

um paciente chegou ao nível superior (30 anos) e todos os demais estão no nível fundamental de estudos; e que dificuldades de aprendizagem e repetência escolar foram detectadas em $55 \%$ dos pacientes.

100.000 crianças, respectivamente. Já em análise feita por Kanamori et al. (1998) no Japão a partir de 20 hospitais nacionais, foram confirmados 675 casos de DMD, com 666 do gênero masculino e 9 do sexo feminino, Esses dados divergem dos encontrados no presente estudo, por não apresentarem indivíduos do gênero feminino atendidos no município de Aracaju nos últimos cinco anos. Isso pode ser explicado devido a população desta cidade ser pequena e nem todas as crianças terem sido atendidas nas clínicas de Fisioterapia.

No presente estudo observou-se relação de associação entre idade de início do tratamento com idade de aparecimento do sinal de Gowers, justificada pela procura por tratamento fisioterapêutico após aparecimento de limitações físicas. 0 surgimento do sinal de Gowers foi observado aos 6 anos de idade, na maioria dos casos estudados, de acordo com a Figura 1; confirmando o descrito por Santos, et al. (2001), que verificou maior frequência entre as idades de 6 a 8 anos.

Segundo Borges (2005), as atividades funcionais dependem do controle de tronco como base 
para o movimento e o tronco deve promover ao mesmo tempo estabilidade e mobilidade para que um indivíduo possa realizar a marcha e suas atividades manuais. A perda do controle de tronco em DMD ocorre devido ao comprometimento da musculatura proximal, dificultando de maneira significativa na musculatura axial. Contrapondo com os achados da pesquisa, embora todos os indivíduos apresentassem encurtamento muscular, em apenas um dos casos a falta de controle de tronco foi observada. Isso mostra a necessidade de elaboração de um programa de intervenção fisioterapêutica, priorizando exercícios que mantenham ou estimulem o controle de tronco em sedestação ou posição ortostática (SANTOS et al., 2009).

Estima-se que metade das crianças adquira marcha independente até os dezoito meses de idade. Por mais que existam variações na evolução do quadro clínico, normalmente, os afetados por DMD não são capazes de andar após os dezesseis anos. A mesma média de idade é confirmada por Otsuka et al. (2005) e Behrman et al. (2003), relatando que a perda da marcha refere-se ao rápido aumento da fraqueza muscular no início da adolescência, causando contraturas e deformidades nos tecidos moles, principalmente de flexores de quadris, feixe iliotibial e músculos da panturrilha, os quais conduzem o paciente de forma mais precoce ao confinamento na cadeira de rodas. Estes resultados são confirmados pelos dados encontrados na pesquisa, mostrando que a maior freqüência de perda da marcha está entre as faixas etárias de 10 e 11 anos (FACHARDO et al., 2004).

Segundo os dados da Organização Mundial de Saúde (OMS), nascem a cada ano 7,6 bilhões de crianças com uma doença genética, sendo $95 \%$ delas nos países subdesenvolvidos. Não existem estudos que relatem a relação existente entre a con- dição socioeconômica e a DMD, porém, o que pode ser acrescentado é o diagnóstico precoce, indispensável para garantir as famílias um melhor planejamento de vida. A dificuldade para um melhor diagnóstico é justificado por Moreira; Araújo (2009) pela falta de reconhecimento da doença pelos médicos. Em seu estudo, a idade média dos primeiros sintomas foi de 2 anos e 11 meses e idade média do diagnóstico definitivo de 6 anos e 10 meses, confirmando com os dados da presente pesquisa.

As crianças com DMD apresentam qualidade de vida comprometida por possuírem um risco três vezes maior de desenvolver problemas emocionais e psicológicos que as crianças sadias. Frequentes conflitos familiares aumentam a percepção negativa e a depressão reduz 0 círculo de amizades, concordando com os achados do presente estudo. Estes dados podem ser comprovados pelos voluntários da pesquisa, durante a aplicação da ficha de anamnese ao possuírem pouca comunicação verbal (GONÇALVES et al., 2008).

Para Moura (2009), a etiologia do comprometimento mental das crianças com DMD e muitos trabalhos descrevem que o atraso no desenvolvimento neuropsicomotor e a deficiência mental estão preferencialmente associados a alterações genéticas no terço final do gene Xp21. Em seu estudo, relatou que os problemas comportamentais podem afetar os indivíduos com DMD, familiares e seus cuidadores, reduzirem o funcionamento cognitivo e problemas de aprendizagem. Estudo publicado por Santos et al. (2006) revelaram que, aproximadamente $65 \%$ apresentam indícios de atrofia cerebral, $75 \%$ dos casos quociente de inteligência (QI) abaixo de 75 e $30 \%$ a $50 \%$ das crianças possuem um quadro de retardo mental moderado ou grave, concordando com o presente estudo, onde a maioria dos voluntários apresentaram histórico de repetição escolar (ARAGÃO; FERREIRA, 2009). 


\section{CONCLUSÃO}

Os pacientes portadores da DMD avaliados no município de Aracaju foram do gênero masculino, com idade média de 13 anos de idade; surgimento do sinal de Gowers foi observado aos 6 anos de idade; as alterações funcionais mais freqüente foram encurtamentos musculares e perda da marcha; quanto ao aspecto de escolaridade, aprendizagem e cognição, encontrou-se que $90 \%$ pacientes iniciaram a escolarização entre os $4 \mathrm{e}$ os 7 anos de idade e que dificuldades de aprendi-

\section{REFERÊNCIAS}

ARAGÃO, E.G.R., FERREIRA, L.M. A atuação da fisioterapia respiratória na reabilitação de paciente com Distrofia Muscular de Duchenne: Estudo de caso. Penápolis: Faculdade de São Paulo, 2009. Disponível em: < http://www.fassp.edu.br/uploads/monografias_131.pdf>. Acesso em: 8 de fevereiro de 2011.

BAUMGARTNER, M., RUIZ, D.A. Distrofia Muscular de Duchenne (Revisión Bibliográfica). Revista médica de Costa Rica. v.65, n 586, 2008.

BEHRMAN, R. E.; KLIEGMAN, R. M.; JENSON, H. B. Fundamentos de Nelson tratado de pediatria. 16. ed. Rio de Janeiro: Guanabara Koogan, 2003.

BORGES, D.; MOURA, E.W.; LIMA, E.; SILVA, P.A.C.. FISIOTERAPIA: Aspectos clínicos e práticos da reabilitação. 1. ed. São Paulo: Artes Médicas, 2007.

CAROMANO, F. A. Efeitos fisiológicos de sessão de hidroterapia em crianças portadoras de distrofia muscular de Duchenne. Revista Fisioterapia, v. 5, n. 1, 1998.

DOOLEY, J. GORDAN, K.E.; DODDS, L.; SWEEN, J.M. Duchenne muscular dystrophy a 30- year population based incidence study. Clinic Pediatric. v. 49, n. 2. March, 2010. zagem e repetência escolar foram detectadas em $55 \%$ dos pacientes.

A maioria apresentou dificuldade para a realização das AVD's, que denota a presença de algumas limitações físicas funcionais, as quais demandam suporte familiar e financeiro adicional, caracterizando a necessidade de implantação de ações e políticas privadas e públicas voltadas para melhoria na qualidade de vida desses pacientes.

FACHARDO, G. A. Tratamento fisioterápico na distrofia muscular de Duchenne: Relato de Caso. Revista Neurociências, v.12, n. 4, 2004.

FREZZA, R. M.; SILVA, S. R.,; FAGUNDES, S. L. Atualização do tratamento fisioterapêutico das distrofias musculares de Duchenne e Becker. RBPS, v. 18, n. 1, 2005.

GONÇALVES, M.; DYLEWSKI, V.; CHAVES, A.C.X.; SILVA, T.M.; FÁVERO, F.M.; FONTES, S.V.; CAMPOS, M.F.C.R.; OLIVEIRA, A.S.B. Qualidade de vida: Análise comparativa entre crianças com Distrofia Muscular de duchenne e seus cuidadores. Revista Neurociências, v. 16, n. 4, 2008.

GYPEL, S.; DAMENT, A. Neurologia Infantil. 3. ed. Belo Horizonte: Atheneu, 1996. IWABE, C., MIRANDA, B.H., NUCCI, A. Medida da função motora: versão da escala para o português e estudo de confiabilidade. Revista Brasileira de Fisioterapia, v.12, n.5, 2008.

KANAMORI, M. Genetic epidemiology of Duchenne Muscular Dystrophy in Japan. Hokkaido Igaku Zasshi, v. 63, n. 6, 1988. 
MACHADO, M. J. da F. Distrofia muscular de duchenne: complicações respiratórias e seu tratamento. $\mathbf{R e}$ vista Ciências Médicas, v. 16, n. 2, 2007.

MARTINEZ, J. A. B.; BRUNHEROTTI, M. A.; ASSIS, M. R.; SOBREIRA, C. F. R. Validação da escala motora funcional EK para a língua portuguesa. Rev. Assoc. Med. Bras., v. 52, n. 5, p. 347-351, 2006.

MOREIRA, A.S.S., ARAÚJO, A.P.Q.C. Não reconhecimento dos sintomas iniciais na atenção primária e a demora do diagnóstico da Distrofia Muscular de Duchenne. Revista Brasileira de Neurologia, v.45, n. 3, 2009.

MOURA, M.C.D.S. Alterações atencionais na Distrofia Muscular de Duchenne. São Paulo: Universidade de São Paulo, São Paulo, 2009. Disponível em: <http://www.teses.usp.br/teses/ disponiveis/47/47135/tde-31072009-15135. php> Acesso em: 23 mar. 2011.

NAIR S. K. P., VASANTH, A.; GOURIE, D.M.; TALY, A.B.; RAO, S.; GAYATHRI, N.; MURALI, T. Disabilities in children with Duchenne muscular dystrophy: a profile. Journal Rehabilitation Medicine, v.33, 2001.

OKAMA, L.O; QUEIROZ, P.D.; SPINA, L.R.; MIRANDA, N.B.L.; CURTARELLI, M.B.; JUNIOR, M.F.; SOUZA, L.A.P.S.. Avaliação funcional e postural nas distrofias de Duchenne e Becker. Revista ConSciencia e Saúde. v.9, n.4, 2010.

OMS, Organização Mundial de Saúde. Disponível em: < http://www.who.int/en/> Acesso em: 21 fev. 2011.

OTSUKA, M. A.; BOFFA, C.F.B.; VIEIRA, A.B.A.D.M. Distrofias Musculares: Fisioterapia Aplicada. 2. ed. São Paulo: Revinter, 2005.

OVANDO, A. C. A Hidroterapia como forma de tratamento para a distrofia muscular de duchenne: Relato de caso. Revista Digital, v 13, n. 126, 2008.
PARREIRA, S.L.S. Quantificação da força muscular e habilidades motoras de pacientes com Distrofia Muscular de Duchenne, em corticoterapia, por período de 1 a 7 anos. São Paulo:Universidade de São Paulo, 2010. Disponível em: <http://www.teses.usp. br/teses/disponiveis/5/5138/tde28052010114357/ en. php> Acesso em: 21 fev. 2011.

PETERLIN, B.; ZIDAR, J.; MEZNARIC, P.M.; ZUPANCIC, N.. Genetic epidemiology in Duchenne and Becker muscular dystrophy in Slovenia. Clinic Genetic, march, v. 51, n. 2, 1997.

REED, U. C.. Doenças neuromusculares. Jornal de Pediatria. v. 78, n.1., 2009.

RODRIGUES, F. Análise do comprometimento pneumofuncional e das habilidades motoras durantes 4 anos de avaliação em paciente com Distrofia Muscular de Duchenne: um estudo de caso. Santa Catarina: Universidade do Extremo Sul Catarinense, 2009. Disponível em: < http://www.bib.unesc.net/biblioteca/ sumario/000042/00004218.pdf> Acesso em: 29 jan. 2011.

SANTOS, N. M.; REZENDE, M.M.; TERNI, A.; HAYASHI, M.C.B.; FÁVERO, F.M.; QUADROS, A.A.J.; REIS, L.I.O.; ADISSI, M.; LANGER, A.L.; FONTES, S.V.; OLIVEIRA, A.S.B. Perfil clínico e funcional dos pacientes com Distrofia Muscular de Duchenne assistidos na associação brasileira de distrofia muscular (ABDIM). Revista Neurociências, v. 14, n.1, 2006.

SILVA, E.C. Análise da função motora de pacientes com Distrofia Muscular de Duchenne em corticoterapia através da escala MFM. São Paulo: Universidade de São Paulo, 2010. Disponível em: < http://www.teses.usp.br/teses/disponiveis/5/5138/ tde11112010093054/es.php> Acesso em: 3 abr. 2011. 


\section{ANEXOE APÊNDICE}

\section{APÊNDICE A - FICHA DE ANAMNESE}

FICHA DE ANAMNESE

Nome completo:

Idade:

Cor:

Nome do pai ou mãe:

Idade:

Renda familiar:

Casa própria: sim () não ()

O voluntário frequenta escola: sim () não () Qual a série:

O voluntário frequenta ensino superior sim ( ) não ( ) Curso e período:

Quando iniciou o processo de escolarização:

Apresenta dificuldades de aprendizagem?

Tem história de repetência escolar?

Deambula: sim () não ()

Idade de aparecimento do sinal de Gowers:

Idade de perda da marcha:

Controle de tronco: ( ) sim ( ) não

Apresenta encurtamentos: ( ) sim ( ) não

Possui cadeira de rodas: sim () não ()

A cadeira de rodas é adaptada: $\operatorname{sim}($ ) não ()

Atividades de lazer:

Possui amigos:

\section{ANEXO A - ESCALA EGEN KLASSIFIKATION \\ ANEXO-ESCALA EK}

\section{Habilidade para o uso da cadeira de rodas}

0 . Capaz de usar uma cadeira de rodas manual no plano, progredindo pelo menos 10 metros em menos de um minuto.

1. Capaz de usar uma cadeira de rodas manual no plano, progredindo 10 metros em mais de um minuto.

2. Incapaz de usar uma cadeira de rodas manual, necessitando de uma cadeira de rodas elétrica.

3. Faz uso de uma cadeira elétrica, mas ocasionalmente apresenta dificuldades para realizar curvas.

\section{Habilidade de transferência de cadeira de rodas}

0 . Capaz de sair da cadeira de rodas sem ajuda.

1. Capaz de sair da cadeira de rodas de maneira independente, mas com necessidade de dispositivo auxiliar.

2. Necessita assistência para sair da cadeira de rodas, com ou sem uso de dispositivos auxiliares.

3. Necessita ser levantado com suporte da cabeça quando saindo da cadeira de rodas. 


\section{Habilidade de permanecer em pé}

0 . Capaz de manter-se em pé com os joelhos apoiados, assim como quando usando muletas.

1. Capaz de manter-se em pé com joelhos e quadris apoiados, assim como quando utilizando dispositivos auxiliares.

2. Capaz de manter-se em pé com apoio para todo o corpo.

3. Incapaz de ficar em pé; presença de contraturas acentuadas.

\section{Habilidade de balançar-se na cadeira de rodas}

0 . Capaz de assumir sozinho uma posição vertical a partir de uma flexão ventral completa com a ajuda das mãos.

1. Capaz de mover a porção superior do corpo mais do que 30 graus a partir da posição sentada em todas as direções, mas incapaz de assumir sem ajuda a

posição vertical a partir de uma flexão ventral completa.

2. Capaz de mover a porção superior do corpo menos de 30 graus de um lado a outro.

3. Incapaz de modificar a posição da parte superior do corpo; não consegue sentar sem o apoio total do tronco e cabeça.

\section{Habilidade de movimentação dos braços.}

0 . Capaz de elevar os braços acima da cabeça com ou sem movimentos compensatórios.

1. Incapaz de levantar os braços acima da cabeça, mas capaz de elevar os antebraços contra a gravidade como, por exemplo, a mão até a boca, com ou sem apoio dos cotovelos.

2. Incapaz de elevar os antebraços contra a gravidade, mas capaz de usar as mãos contra a gravidade quando o antebraço está apoiado.

3. Incapaz de mover as mãos contra a gravidade, mas capaz de usar os dedos.

\section{Habilidade de usar as mãos e braços para comer}

0 . Capaz de cortar a carne em pedaços e comer com colher e garfo. Pode elevar uma tigela cheia (aproximadamente $250 \mathrm{ml}$ ) até a boca sem o apoio do cotovelo.

1. Come e bebe com o cotovelo apoiado.

2. Como e bebe com o cotovelo apoiado e com a ajuda da mão oposta com ou sem a utilização de dispositivos auxiliares alimentares.

3. Necessita ser alimentado.

\section{Habilidade de virar-se na cama}

0 . Capaz de virar-se na cama com as roupas de cama.

1. Capaz de virar-se em um divã, mas não na cama.

2. Incapaz de virar-se na cama. Tem que ser virado três vezes ou menos durante a noite.

3. Incapaz de virar-se na cama. Tem que ser virado quatro vezes ou mais durante a noite.

\section{Habilidade para tossir}

0 . Capaz de tossir efetivamente.

1. Tem dificuldade para tossir e algumas vezes necessita de estímulo manual. Capaz de "limpar a garganta".

2. Sempre necessita de ajuda para tossir. Capaz de tossir somente em certas posições.

3. Incapaz de tossir. Necessita de técnicas de sucção e/ou hiperventilação ou ainda com pressão positiva intermitente para manter as vias aéreas limpas. 


\section{Habilidade para falar}

0 . Fala poderosa. Capaz de cantar e falar alto.

1. Fala normalmente, mas não consegue elevar a sua voz.

2. Fala com voz baixa e precisa respirar após três a cinco palavras.

3. Fala difícil de ser compreendida a não ser pelos parentes próximos.

\section{Bem-estar geral}

0 . Sem queixas, sente-se bem.

1. Cansa facilmente. Apresenta dificuldades quando repousando em uma cadeira ou na cama.

2. Apresenta perda de peso, perda do apetite. Medo de dormir à noite, dorme mal.

3. Apresenta sintomas adicionais tais como: mudanças de humor, dor de estômago, palpitações, sudorese. 